\title{
HOMOGENEOUS SOLUTIONS OF THE GENERALIZED HEAT EQUATION
}

\author{
E. KOCHNEFF
}

(Received 3 March 1995)

Communicated by P. C. Fenton

\begin{abstract}
We discuss expansions of solutions of the generalized heat equation which have a singularity at zero in terms of two sequences of homogeneous solutions.
\end{abstract}

1991 Mathematics subject classification (Amer. Math. Soc.): 35K05, 42 C99.

\section{Introduction.}

A solution of the $n$-dimensional heat equation

$$
\begin{gathered}
\frac{\partial}{\partial t} u(x, t)=\Delta u(x, t)=\sum_{j=0}^{n} \frac{\partial^{2}}{\partial x_{j}^{2}} u(x, t), \\
x=\left(x_{1}, \ldots, x_{n}\right) \in R^{n}, \quad t>0,
\end{gathered}
$$

is called homogeneous of degree $m$ if for all $\lambda>0, x \in R^{n}$ and $t>0$ we have

$$
u\left(\lambda x, \lambda^{2} t\right)=\lambda^{m} u(x, t) .
$$

As part of a program outlining analogies between temperature functions and analytic functions, Rosenbloom and Widder [9] introduced two sequences of temperature functions homogeneous of integer degree. Their first sequence, the 'heat polynomials' homogeneous of degree $m$, are defined by:

$$
v_{m}(x, t)=(-2 t)^{m / 2} H_{m}(x / \sqrt{-2 t}), \quad m=0,1, \ldots
$$

(C) 1997 Australian Mathematical Society $0263-6115 / 97 \$ A 2.00+0.00$ 
where $H_{m}(x)$ is the $m$ th Hermite polynomial orthogonal on $R^{1}$ with respect to $e^{-x^{2} / 2} d x$. These may be considered as an analogue of $\left\{z^{m}\right\}_{m=0}^{\infty}$ for analytic functions.

The natural region of convergence for an expansion in terms of the $\left\{v_{m}\right\}$ is a time strip $|t|<\sigma$. Furthermore, a temperature function $u(x, t)$ has an expansion in terms of the $v_{m}$ valid for $|t|<\sigma$ if and only if the Huygens property holds there: in other words,

$$
u(x, t)=\int_{-\infty}^{\infty} k\left(x-y, t-t^{\prime}\right) u\left(y, t^{\prime}\right) d y, \quad-\sigma<t^{\prime}<t<\sigma
$$

where $k(x, t)$ is the heat kernel:

$$
k(x, t)=\frac{1}{\sqrt{4 \pi t}} e^{-x^{2} / 4 t} .
$$

The 'associated functions' homogeneous of degree $-m-1$, are defined using the Appell transform:

$$
w_{m}(x, t)=\mathscr{A} v_{m}(x, t)=k(x, t) v_{m}(x / t,-1 / t), \quad m=0,1, \ldots
$$

Rosenbloom and Widder showed that a temperature function has an expansion in terms of the $\left\{w_{m}\right\}$ valid for $t>\sigma$ if and only if it satisfies the Huygens principle for $t>\sigma$ and satisfies an additional integrability condition.

On the other hand, the Appell transform maps solutions of the heat equation in $|t|<1 / \sigma$ into solutions of the heat equation in $|t|>\sigma$. In addition, a temperature function satisfies the Huygens property in $|t|<1 / \sigma$ if and only if its Appell transform satisfies a Huygens property in $|t|>\sigma$; that is,

$$
u(x, t)=\int_{R} k\left(x-y, t-t^{\prime}\right) u\left(y, t^{\prime}\right) d y
$$

whenever $t^{\prime}<t<-\sigma, \sigma<t^{\prime}<t$ or both $t^{\prime}>\sigma$ and $t<-\sigma$. Thus expansions in terms of the $\left\{w_{m}\right\}$ are valid in time domains $|t|>\sigma$, and a function $u(x, t)$ can be expanded in terms of the $\left\{w_{m}\right\}$ if and only if $u(x, t)$ satisfies the Huygens property in $|t|>\sigma:$ see [6].

In a continuation of Widder's program, two related papers $[1,4]$ concerned expansions in homogenous solutions of the generalized heat equation

$$
\frac{\partial}{\partial t} u(r, t)=\Delta_{\mu} u(r, t)=\frac{\partial^{2} u}{\partial r^{2}}+\frac{\mu-1}{r} \frac{\partial u}{\partial r}, \quad r>0, \mu>1 .
$$

If $\mu=n$ is an integer, then $\Delta_{\mu}$ is the Laplacian in radial coordinates in $R^{n}$.

Both authors considered expansions in terms of two basic sequences. The radial heat polynomials homogeneous of degree $2 m$ were defined by:

$$
R_{m}^{\mu}(r, t)=m !(4 t)^{m} L_{m}^{(\mu-2) / 2}\left(\frac{-r^{2}}{4 t}\right), \quad m=0,1, \ldots
$$


where $\left\{L_{m}^{(\mu-2) / 2}(x)\right\}$ are the Laguerre polynomials orthogonal with respect to $x^{(\mu-2) / 2} e^{-x} d x$ on $(0, \infty)$.

The associated functions homogeneous of degree $-2 m-1$ are obtained using the Appell transform:

$$
\tilde{R}_{m}^{\mu}(r, t)=\mathscr{A}_{\mu} R_{m}^{\mu}(r, t)=k_{\mu}(r, t) R_{m}^{\mu}(r / t,-1 / t)
$$

where $k_{\mu}$ is the 'fundamental source solution':

$$
k_{\mu}(r, t)=(4 \pi t)^{-\mu / 2} e^{-r^{2} / 4 t} .
$$

Expansion theory for $\left\{R_{m}^{\mu}\right\}$ and $\left\{\tilde{R}_{m}^{\mu}\right\}$ mirrors expansion theory for $\left\{v_{m}\right\}$ and $\left\{w_{m}\right\}$.

In this case the kernel in the Huygens principle is related to the radialization of translations of the heat kemel in $R^{n}$. Let $u\left(x_{1}, x_{2}, \ldots, x_{n}, t\right)$ be a function defined on $R^{n} \times(a, b)$ which is radial in $x=\left(x_{1}, x_{2}, \ldots, x_{n}\right)$, satisfies (1.1) and satisfies the Huygens principle in $R^{n}$. In other words,

$$
u(x, t)=\int_{R^{n}} u\left(y, t^{\prime}\right) k\left(x-y, t-t^{\prime}\right) d y, \quad a<t^{\prime}<t<b,
$$

where $k(x, t)$ is the heat kernel in $R^{n}$

$$
k(x, t)=\frac{1}{(4 \pi t)^{n / 2}} e^{-|x|^{2} / 4 t}, \quad|x|^{2}=x_{1}^{2}+\cdots+x_{n}^{2} .
$$

Let $U(r, t)=u\left(x_{1}, x_{2}, \ldots, x_{n}, t\right), r=|x|$. Then $U(r, t)$ is a solution of $(1.8)$ with $\mu=n$, and switching to polar coordinates with $|y|=\rho, y^{\prime}=y / \rho$ and $\Sigma_{n-1}$ the unit sphere in $R^{n}$ we have

$$
\begin{aligned}
U(r, t) & =\int_{0}^{\infty}\left(\int_{\Sigma_{n-1}} U\left(\rho, t^{\prime}\right) k\left(x-\rho y^{\prime}, t-t^{\prime}\right) d y^{\prime}\right) \rho^{n-1} d \rho \\
& =\int_{0}^{\infty} U\left(\rho, t^{\prime}\right) K_{n}\left(r, \rho, t-t^{\prime}\right) \rho^{n-1} d \rho
\end{aligned}
$$

where

$$
\begin{aligned}
K_{n}(r, \rho, t) & =\int_{\Sigma_{n-1}} k\left(x-\rho y^{\prime}, t\right) d y^{\prime} \\
& =\frac{(r \rho)^{(2-n) / 2}}{2 t} e^{-\left(r^{2}+\rho^{2}\right) / 4 t} I_{(n-2) / 2}\left(\frac{r \rho}{2 t}\right)
\end{aligned}
$$

and $I_{v}(z)$ is the Bessel function of complex argument of order $v$ (see [1]). 
Therefore, for arbitrary $\mu>1$, the Huygens property in $(a, b)$ is defined by

$$
u(r, t)=\int_{0}^{\infty} u\left(\rho, t^{\prime}\right) K_{\mu}\left(r, \rho, t-t^{\prime}\right) \rho^{\mu-1} d \rho, \quad a<t^{\prime}<t<b
$$

where

$$
K_{\mu}(r, \rho, t)=\frac{(r \rho)^{(2-\mu) / 2}}{2 t} e^{-\left(r^{2}+\rho^{2}\right) / 4 t} I_{(\mu-2) / 2}\left(\frac{r \rho}{2 t}\right) .
$$

In [1, 4], it was shown that a function $u(r, t)$ had an expansion in terms of $R_{m}^{\mu}(r, t)$ converging in a strip $|t|<\sigma$ if and only if $u(r, t)$ satisfied the Huygen's property in that strip. Further, it was shown that a function had an expansion in terms of $\tilde{R}_{m}^{\mu}(r, t)$ converging in a domain $t>\sigma$ if and only if it satisfied the Huygen's principle in $t>\sigma$ and satisfied an additional integrability condition.

For $\mu=1,\left\{R_{m}^{\mu}\right\}$ and $\left\{\tilde{R_{m}^{\mu}}\right\}$ reduce to the sequences $\left\{v_{2 m}\right\}_{m=0}^{\infty},\left\{w_{2 m}\right\}_{m=0}^{\infty}$ in [8] because of the connection between the Hermite and Laguerre polynomials. Thus $[1,4]$ included part of the theory of Rosenbloom and Widder.

On the other hand, many elementary solutions of (1.8) cannot be expanded in terms of the radial heat polynomials or their associated functions. All such solutions were entire funcions of $r^{2}$. This precludes expansions for solutions which have a singularity at zero, for example, $r^{2-\mu}$.

However, as we will see below, the approach used in [1] to derive the heat polynomials leads to two other linearly independent sequences of homogenous solutions. We define solutions homogeneous of degree $2 m+2-\mu$ by:

$$
V_{m}^{\mu}(r, t)=r^{2-\mu}(4 t)^{m} m ! L_{m}^{(2-\mu) / 2}\left(\frac{-r^{2}}{4 t}\right), \quad m=0,1, \ldots,
$$

where the $L_{m}^{(2-\mu) / 2}$ are the Laguerre polynomials of order $(2-\mu) / 2$. Note that for $\mu>4$, these polynomials do not form an orthogonal system in the traditional sense.

The associated functions are obtained using the Appell transform:

$$
\tilde{V}_{m}^{\mu}(r, t)=\mathscr{A}_{\mu} V_{m}^{\mu}(r, t)=k_{\mu}(r, t) V_{m}^{\mu}\left(\frac{r}{t}, \frac{-1}{t}\right), \quad m=0,1, \ldots
$$

These are homogenous of degree $-2 m-2$.

For $\mu=1$, the sequences $\left\{V_{m}^{\mu}\right\}$ and $\left\{\tilde{V}_{m}^{\mu}\right\}$ reduce to the sequences $\left\{v_{2 m+1}\right\}_{m=0}^{\infty}$, $\left\{w_{2 m+1}\right\}_{m=0}^{\infty}$ of Rosenbloom and Widder ([8]).

We will show that the natural region of convergence for expansions of $V_{m}^{\mu}$ are time strips $|t|<\sigma$ and that expandibility is equivalent to a certain Huygens principle. We will also prove the corresponding results for the associated functions $\tilde{V}_{m}^{\mu}$. Our main difficulty is working with nonintegrable functions. 
Another aspect of Widder's program was a characterization of all homogeneous solutions of integer degree. The underlying observation is that analytic functions have two linearly independent solutions of degree $n$ for each $n$, namely $\operatorname{Re}\left(z^{n}\right)$ and $\operatorname{Im}\left(z^{n}\right)$. This aspect was extended to (1.8) in [5], and this paper overlaps that one to some extent. Specifically, if $\mu=2,3, \ldots, 2 m+2-\mu \geq 0$, then our $V_{m}^{\mu}, \tilde{V}_{m}^{\mu}$ may be expressed as linear combinations of solutions in [5]. However, we characterize expansions in terms of a Huygens property, while the characterization in [5] is of a function theoretic nature.

We organize the paper as follows. In Sections 2-4 we develop tools needed later in the paper. In Sections 5-7 we derive our homogeneous solutions and discuss their elementary properties including regions of convergence for expansions. Finally, in Sections $8-10$ we discuss Poisson integrals and characterize expansions in terms of the Huygens property.

\section{Hadamard's 'finite part' integral}

To handle divergent integrals, we will use Hadamard's 'finite part' integral.

DEFINITION 2.1. ([3]) Let $n$ be a positive integer, $-(n+1) \leq v<-n$. Let $f(x)$ be a given function, and suppose there exists a polynomial $P_{n-1}(x)$ of degree at most $n-1$ so that

$$
\int_{0}^{\infty}\left|f(x)-P_{n-1}(x)\right| x^{\nu} d x<\infty .
$$

Then Hadamard's finite part (f.p.) integral is defined by

$$
\text { f.p. } \int_{0}^{\infty} f(x) x^{\nu} d x=\int_{0}^{\infty}\left(f(x)-P_{n-1}(x)\right) x^{\nu} d x .
$$

Clearly, if such a polynomial exists, then it is unique. Furthermore, for $-(n+$ $1)<v<-n$, if $f(x), f^{\prime}(x), \ldots, f^{(n)}(x)$ are defined on $[0, a]$ for some $a>0$, if $f^{(n)}(x) \in L\left([0, a] ; x^{\nu+n} d x\right)$ and if $f(x) \in L\left((a, \infty) ; x^{v} d x\right)$ then Hadamard's integral will exist with $P_{n-1}$ the $(n-1)^{\text {st }}$ Taylor polynomial of $f$ centered at 0 . Note that in general Hadamard's integral will not exist if $v=-(n+1)$ unless $f^{(n)}(0)=0$.

For future reference, note that if the first integral below exists, then

$$
\text { f.p. } \int_{0}^{\infty} f(x) x^{(\nu-1) / 2} d x=2 \text { f.p. } \int_{0}^{\infty} f\left(x^{2}\right) x^{\nu} d x .
$$

For $v>-1$, we adopt the convention

$$
\text { f.p. } \int_{0}^{\infty} f(x) x^{\nu} d x=\int_{0}^{\infty} f(x) x^{\nu} d x
$$

Frequently one is able to replace Hadamard's integral with an ordinary integral. 
LEMMA 2.2. Let $n$ be a positive integer, $-(n+1)<v<-n$ and suppose $f(x)$ is $n$-times differentiable on $[0, \infty)$. Then if $f \in L\left((1, \infty) ; x^{\nu} d x\right)$ and $f^{(n)} \in$ $L\left((0, \infty) ; x^{n+v} d x\right)$ we have

$$
\text { f.p. } \int_{0}^{\infty} f(x) x^{\nu} d x=\frac{\Gamma(-n-v)}{\Gamma(-v)} \int_{0}^{\infty} f^{(n)}(x) x^{\nu+n} d x .
$$

PROOF. Let $P_{n-1}$ denote the $(n-1)$ st Taylor polynomial of $f$ centered at 0 . We have

$$
\text { f.p. } \begin{aligned}
\int_{0}^{\infty} f(y) y^{v} d y & =\int_{0}^{\infty}\left(f(y)-P_{n-1}(y)\right) y^{\nu} d y \\
& =\int_{0}^{\infty}\left(\int_{0}^{y} \frac{f^{(n)}(t)}{(n-1) !}(y-t)^{n-1} d t\right) y^{v} d y \\
& =\frac{1}{(n-1) !} \int_{0}^{\infty}\left(\int_{t}^{\infty}(y-t)^{n-1} y^{v} d y\right) f^{(n)}(t) d t \\
& =\frac{\Gamma(-n-v)}{\Gamma(-v)} \int_{0}^{\infty} f^{(n)}(y) y^{\nu+n} d y .
\end{aligned}
$$

We now consider iterated integrals.

THEOREM 2.3. Let $-(n+1)<v<-n, a>0$. Suppose $f(x, y)$ has continuous mixed partials up to degree $n$ in each variable in $[0, a] \times[0, a]$,

$$
\begin{gathered}
\int_{a}^{\infty} \int_{a}^{\infty}|f(x, y)|(x y)^{\nu} d x d y<\infty \\
\int_{a}^{\infty}\left|\partial_{y}^{j} f(x, 0)\right| x^{\nu} d x<\infty, \quad j=0,1, \ldots, n-1,
\end{gathered}
$$

and

Suppose also that there exist functions $g(x) \in L\left((a, \infty) ; x^{v} d x\right)$ and $h(y) \in L((a, \infty)$; $\left.y^{v} d y\right), j=0,1, \ldots, n$ such that

$$
\left|\partial_{y}^{n} f(x, y)\right| \leq g(x), \quad \text { for all } y \in[0, a]
$$

and

$$
\left|\partial_{x}^{n} f(x, y)\right| \leq h(y), \quad \text { for all } x \in[0, a]
$$


Then the iterated integrals

$$
\text { f.p. } \int_{0}^{\infty}\left(\text { f.p. } \int_{0}^{\infty} f(x, y) x^{\nu} d x\right) y^{\nu} d y
$$

and

$$
\text { f.p. } \int_{0}^{\infty}\left(\text { f.p. } \int_{0}^{\infty} f(x, y) y^{v} d y\right) x^{v} d x
$$

both exist and are equal.

PROOF. Let

$$
\begin{gathered}
P_{n-1}(x, y)=\sum_{i=0}^{n-1} \frac{\partial_{x}^{i} f(0, y)}{i !} x^{i}, \quad Q_{n-1}(x, y)=\sum_{i=0}^{n-1} \frac{\partial_{y}^{i} f(x, 0)}{i !} y^{i}, \\
R_{n-1}(x, y)=\sum_{i=0}^{n-1} \sum_{j=0}^{n-1} \frac{\partial_{y}^{i} \partial_{x}^{j} f(0,0)}{j ! i !} x^{j} y^{i},
\end{gathered}
$$

and

$$
M=\max \left\{\left|\partial_{y}^{j} \partial_{x}^{i} f(x, y)\right|: 0 \leq y \leq a, 0 \leq x \leq a, 0 \leq j \leq n, 0 \leq i \leq n\right\} .
$$

By symmetry and Fubini's theorem, it suffices to show that

$$
\begin{aligned}
& \text { f.p. } \int_{0}^{\infty}\left(\text { f.p. } \int_{0}^{\infty} f(x, y) x^{\nu} d x\right) y^{\nu} d y \\
& \quad=\int_{0}^{\infty} \int_{0}^{\infty}\left(f(x, y)-P_{n-1}(x, y)-Q_{n-1}(x, y)+R_{n-1}(x, y)\right)(x y)^{v} d x d y
\end{aligned}
$$

where the latter integral converges absolutely. To prove the absolute convergence, we consider separately the four regions $(a, \infty) \times(a, \infty),(a, \infty) \times(0, a),(0, a) \times(a, \infty)$, and $(0, a) \times(0, a)$.

Clearly, (2.5), (2.6) and (2.7) imply that

$$
\int_{a}^{\infty} \int_{a}^{\infty}\left|f(x, y)-P_{n-1}(x, y)-Q_{n-1}(x, y)+R_{n-1}(x, y)\right|(x y)^{\nu} d x d y<\infty .
$$

Secondly, from (2.9) we have

$$
\begin{aligned}
\int_{a}^{\infty} & \int_{0}^{a}\left|f(x, y)-P_{n-1}(x, y)\right|(x y)^{v} d x d y \\
& =\int_{a}^{\infty} \int_{0}^{a}\left|\frac{1}{(n-1) !} \int_{0}^{x} \partial_{t}^{n} f(t, y)(x-t)^{n-1} d t\right|(x y)^{v} d x d y \\
& \leq \frac{1}{n !}\left(\int_{a}^{\infty} h(y) y^{\nu} d y\right)\left(\int_{0}^{a} x^{n+v} d x\right)<\infty .
\end{aligned}
$$


Also, since

$$
R_{n-1}(x, y)=\sum_{i=0}^{n-1} \frac{\partial_{x}^{i} Q_{n-1}(0, y)}{i !} x^{i}
$$

we have

$$
\begin{aligned}
\int_{a}^{\infty} & \int_{0}^{a}\left|Q_{n-1}(x, y)-R_{n-1}(x, y)\right|(x y)^{v} d x d y \\
& =\int_{a}^{\infty} \int_{0}^{a}\left|\frac{1}{(n-1) !} \int_{0}^{x} \partial_{t}^{n} Q_{n-1}(t, y)(x-t)^{n-1} d t\right|(x y)^{v} d x d y \\
& \leq \frac{1}{(n-1) !} \sum_{i=0}^{n-1} \frac{1}{i !} \int_{a}^{\infty} \int_{0}^{a}\left(\int_{0}^{x}\left|\partial_{t}^{n} \partial_{y}^{i} f(t, 0)\right|(x-t)^{n-1} d t\right) x^{\nu} y^{v+i} d x d y \\
& \leq \frac{M}{n !} \sum_{i=0}^{n-1} \frac{1}{i !}\left(\int_{a}^{\infty} y^{i+v} d y\right)\left(\int_{0}^{a} x^{n+v} d x\right)<\infty
\end{aligned}
$$

Therefore,

$$
\int_{a}^{\infty} \int_{0}^{a}\left|f(x, y)-P_{n-1}(x, y)-Q_{n-1}(x, y)+R_{n-1}(x, y)\right|(x y)^{\nu} d x d y<\infty .
$$

The integral over $(0, a) \times(a, \infty)$ is finite by a similar argument. Finally,

$$
\begin{aligned}
& \int_{0}^{a} \int_{0}^{a}\left|f(x, y)-P_{n-1}(x, y)-Q_{n-1}(x, y)+R_{n-1}(x, y)\right|(x y)^{v} d x d y \\
& \quad=\frac{1}{((n-1) !)^{2}} \int_{0}^{a} \int_{0}^{a}\left|\int_{0}^{y}\left(\int_{0}^{x} \partial_{s}^{n} \partial_{t}^{n} f(t, s)(x-t)^{n-1} d t\right)(y-s)^{n-1} d s\right| \\
& \quad \leq \frac{M}{(n !)^{2}} \int_{0}^{a} \int_{0}^{a}(x y)^{n+v} d x d y d y<\infty .
\end{aligned}
$$

This proves the absolute convergence of the integral.

Let

$$
k(y)=\text { f.p. } \int_{0}^{\infty} f(x, y) x^{\nu} d x=\int_{0}^{\infty}\left(f(x, y)-P_{n-1}(x, y)\right) x^{\nu} d x .
$$

By the first hypothesis and Fubini's theorem this integal exists for a.e. $y$. Let

$$
K_{n-1}(y)=\int_{0}^{\infty}\left(Q_{n-1}(x, y)-R_{n-1}(x, y)\right) x^{\nu} d x .
$$


Then $K_{n-1}$ is a polynomial of degree $n-1$ for which

$$
\int_{0}^{\infty}\left|k(y)-K_{n-1}(y)\right| y^{\nu} d y<\infty
$$

Therefore

$$
\begin{aligned}
\text { f.p. } & \int_{0}^{\infty}\left(\text { f.p. } \int_{0}^{\infty} f(x, y) x^{\nu} d x\right) y^{\nu} d y=\int_{0}^{\infty}\left(k(y)-K_{n-1}(y)\right) y^{\nu} d y \\
\quad= & \int_{0}^{\infty} \int_{0}^{\infty}\left(f(x, y)-P_{n-1}(x, y)-Q_{n-1}(x, y)+R_{n-1}(x, y)\right)(x y)^{\nu} d x d y .
\end{aligned}
$$

This completes the proof.

\section{Laguerre polynomials}

For $v>-1$, the Laguerre polynomials $\left\{L_{m}^{v}(x)\right\}$ are defined by orthogonality:

$$
\int_{0}^{\infty} L_{k}^{v}(x) L_{m}^{\nu}(x) e^{-x} x^{v} d x=\frac{\Gamma(k+v+1)}{k !} \delta_{k m}, \quad k, m=0,1,2, \ldots
$$

and the condition that each $L_{m}^{v}(x)$ is a polynomial of degree $m$ with coefficient of $x^{m}$ equal to $(-1)^{m}$. The definition extends to all $v \in C$ using the explicit representation:

$$
L_{m}^{\nu}(x)=\sum_{j=0}^{m} \frac{\Gamma(m+v+1)}{\Gamma(j+v+1)} \frac{(-1)^{j} x^{j}}{j !(m-j) !}, \quad m=0,1, \ldots
$$

or:

$$
L_{m}^{\nu}(x)=\frac{e^{x} x^{-v}}{m !} \frac{d^{m}}{d x^{m}}\left(e^{-x} x^{v+m}\right) .
$$

Orthogonality extends to Laguerre polynomials of negative order $v \neq-1,-2, \ldots$ using Hadamard's integral ([6]):

$$
\text { f.p. } \int_{0}^{\infty} L_{k}^{\nu}(x) L_{m}^{v}(x) e^{-x} x^{\nu} d x=\frac{\Gamma(k+v+1)}{k !} \delta_{k, m}, \quad k, m=0,1,2, \ldots
$$

For $v=-1,-2, \ldots$ the orthogonality relation holds for restricted indices. Let $v=-l$, where $l$ denotes a positive integer. Then for all $k \geq l[10]$ :

$$
L_{k}^{(-l)}(x)=(-x)^{l} \frac{(k-l) !}{k !} L_{k-l}^{(l)}(x)
$$

so that we have for $k, m \geq l$ :

$$
\begin{aligned}
\int_{0}^{\infty} L_{k}^{(-l)}(x) L_{m}^{(-l)}(x) e^{-x} x^{-l} d x & =\frac{(k-l) !(m-l) !}{k ! m !} \int_{0}^{\infty} L_{k-l}^{(l)}(x) L_{m-l}^{(l)}(x) e^{-x} x^{l} d x \\
& =\frac{(k-l) !}{k !} \delta_{k, m} .
\end{aligned}
$$




\section{Laguerre polynomials: integral formulae, inequalities, and expansions}

It is known that for all $x \in C, m+v>-1$ ([10]):

$$
L_{m}^{v}(x)=\frac{e^{x} x^{-v / 2}}{m !} \int_{0}^{\infty} s^{m+v / 2} J_{v}(2 \sqrt{x s}) e^{-s} d s
$$

where $J_{v}(z)$ is the Bessel function of order $v$ :

$$
J_{v}(z)=\sum_{k=0}^{\infty} \frac{(-1)^{k}(z / 2)^{v+2 k}}{k ! \Gamma(k+v+1)} .
$$

The Bessel function of complex argument is defined by:

$$
I_{\nu}(z)=i^{-v} J_{\nu}(-i z)=\sum_{k=0}^{\infty} \frac{(z / 2)^{\nu+2 k}}{k ! \Gamma(k+v+1)} .
$$

For $-\pi / 2+\delta \leq \arg (z) \leq 3 \pi / 2-\delta, \delta>0$, the following assymptotic formula holds [9]:

$$
I_{\nu}(z)=(2 \pi z)^{-1 / 2}\left(e^{z}+e^{-z+\left(v+\frac{1}{2}\right) \pi i}\right)\left\{1+O\left(|z|^{-1}\right)\right\}, \quad z \rightarrow \infty .
$$

Define

$$
I_{\nu}^{*}(z)=z^{-v / 2} I_{\nu}(2 \sqrt{z})=\sum_{k=0}^{\infty} \frac{z^{k}}{k ! \Gamma(k+\nu+1)}, \quad z \in C .
$$

Then for $-\pi / 2+\delta \leq \arg (z) \leq 3 \pi / 2-\delta, \delta>0$ :

$$
I_{\nu}^{*}(z)=\frac{1}{\sqrt{4 \pi}} z^{-\nu / 2-1 / 4}\left(e^{2 \sqrt{z}}+e^{-2 \sqrt{z}+(\nu+1 / 2) \pi i}\right)\left\{1+O\left(|z|^{-1 / 2}\right)\right\}, \quad z \rightarrow \infty .
$$

THEOREM 4.1. For $m=0,1, \ldots, x \in C, v \in R, m+v \neq-1,-2, \ldots$, we have

$$
m ! L_{m}^{\nu}(-x)=\text { f.p. } \int_{0}^{\infty} I_{\nu}^{*}(x s) e^{-(s+x)} s^{m+v} d s .
$$

PROOF. This is equation (4.1) if $m+v>-1$. Therefore, since the left hand side above is an analytic function of $v$, it suffices to prove that the right hand side is an analytic function of $v$, for $m+v \neq-1,-2, \ldots$ We will do this by showing that for all $x \in C$, and $m+v \neq-1,-2, \ldots$ we have

$$
\text { f.p. } \int_{0}^{\infty} s^{m+v} I_{\nu}^{*}(x s) e^{-s} d s=\sum_{k=0}^{\infty} \frac{x^{k} \Gamma(k+v+m+1)}{k ! \Gamma(k+v+1)}
$$


where the last sum converges almost uniformly with repect to $v$. Fix $n$ so that $m+n+v>-1$. Then by (3.4) with $k=m=0$

$$
\Gamma(v+1)=\text { f.p. } \int_{0}^{\infty} s^{v} e^{-s} d s, \quad v \neq-1,-2, \ldots,
$$

so that formally

$$
\begin{aligned}
\text { f.p. } \int_{0}^{\infty} s^{m+v} I_{v}^{*}(x s) e^{-s} d s= & \sum_{k=0}^{n-1} \frac{x^{k}}{k ! \Gamma(k+v+1)} \text { f.p. } \int_{0}^{\infty} s^{m+v+k} e^{-s} d s \\
& +\int_{0}^{\infty} s^{m+v} \sum_{k=n}^{\infty} \frac{(x s)^{k}}{k ! \Gamma(k+v+1)} e^{-s} d s \\
= & \sum_{k=0}^{\infty} \frac{x^{k} \Gamma(k+v+m+1)}{k ! \Gamma(k+v+1)}
\end{aligned}
$$

The exchange of integration and summation is justified, since for fixed $\nu, m$,

$$
\frac{\Gamma(m+k+v+1)}{\Gamma(k+v+1)}=O\left(k^{m}\right), \quad k \rightarrow \infty .
$$

This completes the proof.

THEOREM 4.2. For all $x \in C, v \in R, a \neq 1, m=0,1, \ldots, m+v \neq-1,-2, \ldots$, we have

$$
L_{m}^{\nu}\left(\frac{a x}{1-a}\right)=\frac{1}{(1-a)^{m}} \text { f.p. } \int_{0}^{\infty} I_{\nu}^{*}(x y) L_{m}^{\nu}(a y) e^{-(x+y)} y^{\nu} d y .
$$

ProOF. The case $v>-1$ is due to Erdelyi ([2]). For $v<-1, v \neq-2,-3, \ldots$ we have, by Theorem 4.1 ,

$$
\begin{aligned}
\text { f.p. } \int_{0}^{\infty} I_{v}^{*}(x y) L_{m}^{v}(a y) e^{-(x+y)} y^{\nu} d y \\
\quad=\sum_{j=0}^{m} \frac{\Gamma(m+v+1)}{\Gamma(j+v+1)} \frac{(-a)^{j}}{j !(m-j) !} \text { f.p. } \int_{0}^{\infty} I_{v}^{*}(x y) e^{-(x+y)} y^{\nu+j} d y \\
=\sum_{j=0}^{m} \frac{\Gamma(m+v+1)}{\Gamma(j+v+1)} \frac{(-a)^{j}}{(m-j) !} L_{j}^{v}(-x) .
\end{aligned}
$$

The last quantity is an entire function of $v$ for each $a$ and $x$ and is equal to $(1-a)^{m} L_{m}^{\nu}(a x /(1-a)$ ) (which is also entire) for $v>-1$; therefore equality holds for all $v$. 
If $v=-k, k=1,2, \ldots$, then $m+v \neq-1,-2, \ldots$ implies $m \geq k$. Since

$$
I_{-k}^{*}(y)=y^{k} I_{k}^{*}(y) \quad \text { and } \quad L_{m}^{(-k)}(y)=(-y)^{k} \frac{(m-k) !}{m !} L_{m-k}^{(k)}(y)
$$

the theorem in this case follows from the theorem for $v=k$.

By multiplying $(1-a)^{m}$ onto the left hand side of equation (4.9) and taking the limit as $a \rightarrow 1$ we obtain

COROLLARY 4.3. For all $x \in C, m=0,1, \ldots, v \in R, m+v \neq-1,-2, \ldots$, we have

$$
\frac{(-x)^{m}}{m !}=\text { f.p. } \int_{0}^{\infty} I_{v}^{*}(x y) e^{-(x+y)} L_{m}^{\nu}(y) y^{\nu} d y
$$

Note that for $v>-1, \gamma \in R$, integrals of the form $\int_{0}^{\infty} I_{\gamma}^{*}(z y) F(y) y^{v} d y$ define analytic functions of $z$. Similarly, for $v<-1$ we have

THEOREM 4.4. Let $0<a<b, \gamma \in R, v<-1$ and $v \neq-2,-3, \ldots$ Suppose

$$
u(z, x)=\text { f.p. } \int_{0}^{\infty} I_{\gamma}^{*}(z y) e^{-x y} F(y) y^{\nu} d y
$$

is well-defined for $x \in(a, b)$ and all $z \in C$. Then $u(z, x)$ is an entire function of $z$ for each $x \in(a, b)$.

PROOF. Fix $x$. Suppose $-(n+1)<v<-n$. We first prove the existence of the integrals

$$
\text { f.p. } \int_{0}^{\infty} e^{-x y} F(y) y^{\nu+j} d y, \quad j=0,1, \ldots
$$

The case $j=0$ follows from the hypothesis with $z=0$.

Let $P_{n-1}(y)=a_{0}+a_{1} y+\cdots+a_{n-1} y^{n-1}$ be the polynomial in the definition of Hadamard's integral for the case $j=0$. For $j=1,2, \ldots, n-1$ let $P_{j-1}(y)=$ $a_{0}+a_{1} y+\cdots+a_{j-1} y^{j-1}$.

If $j \geq n$, then $v+j>-1$, so that

$$
\begin{aligned}
\int_{0}^{1}|F(y)| e^{-x y} y^{\nu+j} d y & \leq \int_{0}^{1}\left|F(y) e^{-x y}-P_{n-1}(y)\right| y^{\nu} d y+\int_{0}^{1}\left|P_{n-1}(y)\right| y^{\nu+j} d y \\
& <\infty
\end{aligned}
$$




$$
\begin{aligned}
& \text { If } j=1,2, \ldots, n-1 \text { then }-(n-j+1)<v+j<-(n-j) \text { and } \\
& \int_{0}^{1}\left|F(y) e^{-x y}-P_{n-j-1}(y)\right| y^{v+j} d y \\
& \quad \leq \int_{0}^{1}\left|F(y) e^{-x y}-P_{n-1}(y)\right| y^{v} d y+\int_{0}^{1}\left|P_{n-1}(y)-P_{n-j-1}(y)\right| y^{v+j} d y<\infty .
\end{aligned}
$$

Since also

$$
\int_{1}^{\infty} e^{-x y}|F(y)| y^{v+j} d y<\infty
$$

for all $j \in N$ and $x \in(a, b)$, this proves the claim.

Define

$$
I_{\gamma, m}^{*}(z)=\sum_{j=m}^{\infty} \frac{z^{j}}{j ! \Gamma(\gamma+j+1)}
$$

where $m$ is chosen sufficiently large so that both $m+\gamma, m+v>-1$. Note that

$$
\begin{aligned}
\left|I_{\gamma, m}^{*}(z)\right| & \leq|z|^{m} \sum_{j=m}^{\infty} \frac{|z|^{j-m}}{j ! \Gamma(\gamma+j+1)} \\
& =|z|^{m} \sum_{j=0}^{\infty} \frac{|z|^{j}}{(j+m) ! \Gamma(\gamma+m+j+1)} \\
& \leq|z|^{m} I_{\gamma+m}^{*}(|z|) .
\end{aligned}
$$

Define

$$
v(z, x)=\int_{0}^{\infty} I_{y, m}^{*}(z y) e^{-x y} F(y) y^{\nu} d y
$$

Fix $\epsilon, M>0$, and suppose $|z| \leq M$. Let $x^{\prime} \in(a, b), x^{\prime}<x$. There exists a constant $C$ independent of $z$ such that

$$
\begin{aligned}
\int_{0}^{\infty}\left|I_{\gamma, m}^{*}(z y) F(y)\right| e^{-x y} y^{\nu} d y & \leq C|z|^{m} \int_{0}^{\infty} e^{(2+\epsilon) \sqrt{y|z|}} e^{-x y}|F(y)| y^{\nu+m} d y \\
& \leq C \int_{0}^{\infty} e^{-x^{\prime} y}|F(y)| y^{\nu+m} d y
\end{aligned}
$$

Let $\rho$ be any closed curve in the region $|z| \leq M$. By Fubini's Theorem we have

$$
\int_{\rho} v(z, x) d z=\int_{0}^{\infty}\left(\int_{\rho} I_{\gamma, m}^{*}(z y) d z\right) e^{-x y} F(y) y^{v} d y=0 .
$$


Therefore, by Morera's Theorem, $v(z, x)$ is entire in $z$. Since

$$
u(z, x)=v(z, x)+\sum_{j=0}^{m-1} \frac{z^{j}}{j ! \Gamma(\gamma+j+1)} \text { f.p. } \int_{0}^{\infty} e^{-x y} F(y) y^{v+j} d y
$$

this completes the proof.

We will need size estimates on the Laguerre polynomials for both positive and negative values of $x$. For positive values of $x$ we have:

LEMMA 4.5. (i) ([10], p. 241) For any $v \in R$ and $c>0$

$$
L_{m}^{\nu}(x)=O\left(m^{\nu / 2-1 / 4}\right) e^{x / 2} x^{-\nu / 2-1 / 4}, \quad m \rightarrow \infty
$$

uniformly in $(c, \infty)$.

(ii) ([10], p. 178) For any $v \in R$ and $w>0$ we have

$$
L_{m}^{\nu}(x)=O\left(m^{\gamma}\right), \quad m \rightarrow \infty
$$

uniformly in $[0, w]$, where $\gamma=\max \{v, v / 2-1 / 4\}$.

For negative values of $x$ we have:

LEMMA 4.6. (i) For any $\delta>1, v \in R$ and $c>0$ we have

$$
\left|L_{m}^{\nu}(-x)\right|=O\left(m^{\nu / 2-1 / 4} \delta^{m}\right) e^{x /(\delta-1)} x^{-\nu / 2-1 / 4}, \quad m \rightarrow \infty
$$

uniformly in $(c, \infty)$.

(ii) For any $\delta>1, v \in R$ and $w>0$ we have

$$
\left|L_{m}^{v}(-x)\right|=O\left(m^{\gamma} \delta^{m}\right), \quad m \rightarrow \infty
$$

uniformly for $x \in[0, w]$, where $\gamma=\max \{\nu, v / 2-1 / 4\}$.

ProOF. (i) From (4.6),

$$
I_{\nu}^{*}(s)=O(1) s^{-\nu / 2-1 / 4} e^{2 \sqrt{s}}, \quad s \in R, s \rightarrow \infty .
$$

Thus, there exists a constant $C$ independent of $x>0$ such that

$$
\left|I_{\nu}^{*}(x s)\right| \leq C \begin{cases}1 & \text { if } s<1 / x \\ (x s)^{-v / 2-1 / 4} e^{2 \sqrt{x s}} & \text { if } s \geq 1 / x\end{cases}
$$


Let $m+v>-1$. By Theorem 4.1

$$
\begin{aligned}
\left|L_{m}^{\nu}(-x)\right| & \leq \frac{1}{m !} \int_{0}^{\infty}\left|I_{\nu}^{*}(x s)\right| e^{-(s+x)} s^{m+v} d s \\
& =\frac{1}{m !}\left\{\int_{0}^{\frac{1}{x}}\left|I_{\nu}^{*}(x s)\right| e^{-(s+x)} s^{m+v} d s+\int_{\frac{1}{x}}^{\infty}\left|I_{\nu}^{*}(x s)\right| e^{-(s+x)} s^{m+v} d s\right\} \\
& \leq \frac{C}{m !}\left\{\int_{0}^{\frac{1}{x}} e^{-(s+x)} s^{m+v} d s+x^{-v / 2-1 / 4} \int_{0}^{\infty} e^{-(\sqrt{s}-\sqrt{x})^{2}} s^{m+v / 2-1 / 4} d s\right\} .
\end{aligned}
$$

Therefore, since for any $\delta>1$ and all $x, s \geq 0$ :

$$
e^{-(\sqrt{s}-\sqrt{x})^{2}} \leq e^{x /(\delta-1)} e^{-s / \delta}
$$

we have uniformly for $x \geq c, c>0$

$$
\begin{aligned}
\left|L_{m}^{\nu}(-x)\right| & \leq \frac{O(1)}{m !} x^{-\nu / 2-1 / 4} e^{x /(\delta-1)} \int_{0}^{\infty} e^{-s / \delta} s^{m+v / 2-1 / 4} d s \\
& =\frac{O(1)}{m !} x^{-\nu / 2-1 / 4} e^{x /(\delta-1)} \delta^{m} \Gamma(m+\nu / 2+3 / 4) \\
& =O\left(m^{\nu / 2-1 / 4} \delta^{m}\right) x^{-v / 2-1 / 4} e^{x /(\delta-1)} .
\end{aligned}
$$

(ii). If $v \geq-1 / 2$, then (4.19) implies that we have $\left|I_{\nu}^{*}(s)\right| \leq C e^{2 \sqrt{s}}, s \geq 0$ so that we have uniformly for $x \in[0, w]$

$$
\begin{aligned}
\left|L_{m}^{v}(-x)\right| & \leq \frac{C}{m !} \int_{0}^{\infty} e^{-(\sqrt{s}-\sqrt{x})^{2}} s^{m+v} d s \\
& \leq \frac{C}{m !} \int_{0}^{\infty} e^{-s / \delta} s^{m+v} d s \\
& =O\left(m^{v} \delta^{m}\right) .
\end{aligned}
$$

If $v<-1 / 2$, then $-v / 2-1 / 4 \geq 0$, so from (4.20) we have uniformly for $x \in[0, w]$

$$
\begin{aligned}
\left|L_{m}^{\nu}(-x)\right| & \leq \frac{C}{m !}\left\{\int_{0}^{\infty} e^{-(s+x)} s^{m+v} d s+\int_{0}^{\infty} e^{-(\sqrt{s}-\sqrt{x})^{2}} s^{m+v / 2-1 / 4} d s\right\} \\
& \leq \frac{C}{m !}\left\{\Gamma(m+v+1)+\delta^{m+v / 2+3 / 4} \Gamma(m+v / 2+3 / 4)\right\} \\
& =O\left(m^{\nu / 2-1 / 4} \delta^{m}\right) .
\end{aligned}
$$

This concludes the proof. 
THEOREM 4.7. For $n \in N,-(n+1)<v<-n$ and $\gamma_{n}=\max \{v+n,(v+n) / 2-$ $1 / 4\}$ suppose

$$
\sum_{m=0}^{\infty}\left|c_{m}\right| m^{\gamma_{n}}<\infty
$$

Let $c>0$ and let $f$ be a function for which

$$
\int_{c}^{\infty}|f(x)| e^{-x / 2} x^{\nu / 2-1 / 4} d x<\infty
$$

and for which

$$
\text { f.p. } \int_{0}^{\infty} f(x) e^{-x} x^{v} d x
$$

exists. Then

$$
\text { f.p. } \int_{0}^{\infty} f(x)\left(\sum_{m=0}^{\infty} c_{m} L_{m}^{\nu}(x)\right) e^{-x} x^{\nu} d x=\sum_{m=0}^{\infty} c_{m} \text { f.p. } \int_{0}^{\infty} f(x) L_{m}^{\nu}(x) e^{-x} x^{\nu} d x .
$$

PROOF. Since $d\left(L_{m}^{\nu}(x)\right) / d x=-L_{m-1}^{\nu+1}(x)$, we have for $j=0,1, \ldots, n$ :

$$
\sum_{m=0}^{\infty}\left|c_{m} \frac{d^{j}}{d x^{j}} L_{m}^{\nu}(x)\right|=O(1) \begin{cases}1 & \text { if } x \in[0, c] \\ e^{x / 2} x^{-(\nu+j) / 2-1 / 4} & \text { otherwise. }\end{cases}
$$

Therefore by Lemma 2.2 the theorem clearly holds if $f$ is sufficiently nice, for example, a polynomial. For more general $f$, since (4.24) exists, there exists a polynomial

$$
Q_{n-1}(x)=a_{0}+a_{1} x+\cdots+a_{n-1} x^{n-1}
$$

such that

$$
\int_{0}^{1}\left|f(x) e^{-x}-Q_{n-1}(x)\right| x^{\nu} d x<\infty
$$

Let $\mathscr{P}_{j}$ denote the $j$ th Taylor polynomial of $e^{x}$ centered at 0 , and let

$$
P_{n-1}(x)=\sum_{j=0}^{n-1} a_{j} x^{j} \mathscr{P}_{n-1-j}(x) .
$$

Then clearly

$$
\int_{0}^{1}\left|f(x)-P_{n-1}(x)\right| x^{\nu} d x<\infty
$$


By orthogonality, we have for $m \geq n$,

$$
\begin{aligned}
& \text { f.p. } \int_{0}^{\infty} f(x) L_{m}^{\nu}(x) e^{-x} x^{\nu} d x \\
& \quad=\int_{0}^{\infty}\left(f(x)-P_{n-1}(x)\right) L_{m}^{\nu}(x) e^{-x} x^{\nu} d x+\text { f.p. } \int_{0}^{\infty} P_{n-1}(x) L_{m}^{\nu}(x) e^{-x} x^{\nu} d x \\
& \quad=\int_{0}^{\infty}\left(f(x)-P_{n-1}(x)\right) L_{m}^{\nu}(x) e^{-x} x^{\nu} d x .
\end{aligned}
$$

Furthermore, for $c>0$, using Lemma 4.5 we have

$$
\begin{aligned}
& \sum_{m=n}^{\infty}\left|c_{m}\right| \int_{0}^{\infty}\left|\left(f(x)-P_{n-1}(x)\right) L_{m}^{\nu}(x)\right| e^{-x} x^{\nu} d x \\
& \leq C \sum_{m=n}^{\infty}\left|c_{m}\right|\left\{m^{\gamma_{n}} \int_{0}^{c}\left|f(x)-P_{n-1}(x)\right| x^{\nu} d x\right. \\
& \left.\quad+m^{\nu / 2-1 / 4} \int_{c}^{\infty}\left|f(x)-P_{n-1}(x)\right| e^{-x / 2} x^{\nu / 2-1 / 4} d x\right\}
\end{aligned}
$$$$
<\infty \text {. }
$$

Therefore,

$$
\begin{aligned}
\sum_{m=n}^{\infty} c_{m} \text { f.p. } \int_{0}^{\infty} f(x) L_{m}^{\nu}(x) e^{-x} x^{\nu} d x \\
=\sum_{m=n}^{\infty} c_{m} \int_{0}^{\infty}\left(f(x)-P_{n-1}(x)\right) L_{m}^{\nu}(x) e^{-x} x^{\nu} d x \\
=\int_{0}^{\infty}\left(f(x)-P_{n-1}(x)\right)\left(\sum_{m=n}^{\infty} c_{m} L_{m}^{\nu}(x)\right) e^{-x} x^{\nu} d x \\
=\text { f.p. } \int_{0}^{\infty} f(x)\left(\sum_{m=n}^{\infty} c_{m} L_{m}^{\nu}(x)\right) e^{-x} x^{\nu} d x \\
\quad-\text { f.p. } \int_{0}^{\infty} P_{n-1}(x)\left(\sum_{m=n}^{\infty} c_{m} L_{m}^{\nu}(x)\right) e^{-x} x^{\nu} d x \\
=\text { f.p. } \int_{0}^{\infty} f(x)\left(\sum_{m=n}^{\infty} c_{m} L_{m}^{\nu}(x)\right) e^{-x} x^{\nu} d x .
\end{aligned}
$$

This proves the theorem.

One application of Theorem 4.7 is: 
THEOREM 4.8. For all $v \in R, x, s \in C$ :

$$
\text { f.p. } \int_{0}^{\infty} I_{v}^{*}(y s) I_{v}^{*}(y x) e^{-y} y^{v} d y=e^{s+x} I_{v}^{*}(x s) .
$$

PROOF. This is well-known if $v>-1$. By multiplying power series we obtain:

$$
e^{-s} I_{\nu}^{*}(y s)=\sum_{m=0}^{\infty} \frac{(-s)^{m}}{\Gamma(m+v+1)} L_{m}^{v}(y) .
$$

Therefore for $v \neq-1,-2, \ldots$, we have, by Theorem 4.7 and Corollary 4.3,

$$
\begin{aligned}
\text { f.p. } \int_{0}^{\infty} I_{v}^{*}(y s) I_{v}^{*}(y x) e^{-y} y^{v} d y \\
=e^{s} \text { f.p. } \int_{0}^{\infty}\left(\sum_{m=0}^{\infty} \frac{(-s)^{m}}{\Gamma(m+v+1)} L_{m}^{v}(y)\right) I_{v}^{*}(y x) e^{-y} y^{v} d y \\
=e^{s} \sum_{m=0}^{\infty} \frac{(-s)^{m}}{\Gamma(m+v+1)} \text { f.p. } \int_{0}^{\infty} L_{m}^{v}(y) I_{v}^{*}(y x) e^{-y} y^{v} d y \\
=e^{s+x} I_{v}^{*}(x s) .
\end{aligned}
$$

For $v=-k, k=1,2, \ldots$, we have

$$
\begin{aligned}
\int_{0}^{\infty} I_{-k}^{*}(y s) I_{-k}^{*}(y x) e^{-y} y^{-k} d y & =(s x)^{k} \int_{0}^{\infty} I_{k}^{*}(y s) I_{k}^{*}(y x) e^{-y} y^{k} d y \\
& =(s x)^{k} e^{s+x} I_{k}^{*}(s x) \\
& =e^{s+x} I_{-k}^{*}(s x) .
\end{aligned}
$$

COROLlARY 4.9. For all $a>0, x, s, v \in R$ :

$$
\text { f.p. } \int_{0}^{\infty} I_{\nu}^{*}(y s) I_{\nu}^{*}(y x) e^{-a y} y^{\nu} d y=a^{-\nu-1} e^{(x+s) / a} I_{\nu}^{*}\left(x s / a^{2}\right) .
$$

\section{Homogeneous solutions}

We consider solutions of equation (1.8) whose boundary values are powers of $r$. Following [1], we formally calculate such solutions:

$$
e^{t \Delta_{\mu}} r^{2 \gamma}=\sum_{k=0}^{\infty} \frac{t^{k}}{k !} \Delta_{\mu}^{k} r^{2 \gamma}=\sum_{k=0}^{\infty} \frac{(4 t)^{k}}{k !} \frac{\Gamma(\gamma+1) \Gamma\left(\gamma+\frac{\mu}{2}\right)}{\Gamma(\gamma+1-k) \Gamma\left(\gamma+\frac{\mu}{2}-k\right)} r^{2 \gamma-2 k}
$$


The series above terminates if $\gamma=m$ or if $\gamma=m+1-\mu / 2, m=0,1, \ldots$, and diverges otherwise. The radial heat polynomials $R_{m}^{\mu}(r, t)$ defined in equation (1.9) are obtained by letting $\gamma=m$. Letting $\gamma=m+1-\mu / 2, m=0,1, \ldots$, we define for $r \neq 0$ :

$$
\begin{aligned}
V_{m}^{\mu}(r, t) & =e^{t \Delta_{\mu}} r^{2 \gamma} \\
& =\sum_{k=0}^{m} \frac{(4 t)^{k}}{k !} \frac{\Gamma\left(m+2-\frac{\mu}{2}\right) m !}{\Gamma\left(m+2-\frac{\mu}{2}-k\right) \Gamma(m-k+1)} r^{2 m+2-\mu-2 k} \\
& =r^{2-\mu} \sum_{k=0}^{m} \frac{(4 t)^{m-k}}{(m-k) !} \frac{\Gamma\left(m+2-\frac{\mu}{2}\right) m !}{\Gamma\left(k+2-\frac{\mu}{2}\right) k !} r^{2 k} \\
& =r^{2-\mu}(4 t)^{m} \sum_{k=0}^{m}\left(\begin{array}{c}
m \\
k
\end{array}\right)\left(\frac{r^{2}}{4 t}\right)^{k} \frac{\Gamma\left(m+2-\frac{\mu}{2}\right)}{\Gamma\left(k+2-\frac{\mu}{2}\right)} \\
& =r^{2-\mu}(4 t)^{m} m ! L_{m}^{(2-\mu) / 2}\left(-r^{2} / 4 t\right) .
\end{aligned}
$$

It is easy to verify that $V_{m}^{\mu}(r, t)$ are solutions of (1.8) which are homogenous of degree $2 m+2-\mu$. Note $V_{0}^{\mu}(r, t)=r^{2-\mu}, V_{m}^{\mu}(r, t)=O\left(r^{2-\mu}\right), r \rightarrow 0$, and $V_{m}^{\mu}(r, 0)=r^{2-\mu+2 m}, m=0,1, \ldots$, except in the case $\mu=2 k+2, m \geq k$.

In the latter case, $V_{m}^{\mu}$ are identical to $R_{m-k}^{\mu}$ :

$$
\begin{aligned}
V_{m}^{\mu}(r, t) & =r^{-2 k}(4 t)^{m} m ! L_{m}^{(-k)}\left(-r^{2} / 4 t\right) \\
& =(4 t)^{m-k}(m-k) ! L_{m-k}^{(k)}\left(-r^{2} / 4 t\right) \\
& =R_{m-k}^{\mu}(r, t) .
\end{aligned}
$$

We define the associated functions using the Appell transform:

$$
\begin{aligned}
\tilde{V}_{m}^{\mu}(r, t) & =k_{\mu}(r, t) V_{m}^{\mu}(r / t,-1 / t) \\
& =k_{\mu}(r, t) t^{\mu-2-2 m} V_{m}^{\mu}(r,-t) \\
& =r^{2-\mu} k_{\mu}(r, t) t^{\mu-2-2 m}(-4 t)^{m} m ! L_{m}^{(2-\mu) / 2}\left(r^{2} / 4 t\right) .
\end{aligned}
$$

The $\tilde{V}_{m}^{\mu}(r, t)$ are homogeneous of degree $-2 m-2$ and $\tilde{V}_{m}^{\mu}(r, t)=O\left(r^{2-\mu}\right), r \rightarrow 0$, except for $\mu=2 k+2, k=0,1, \ldots, m \geq k$, in which case

$$
\tilde{V}_{m}^{\mu}(r, t)=\tilde{R}_{m-k}^{\mu}(r, t) .
$$

For $\mu=1,\left\{V_{m}^{\mu}\right\}$ and $\left\{\tilde{V}_{m}^{\mu}\right\}$ coincide with $\left\{v_{2 m+1}\right\}$ and $\left\{w_{2 m+1}\right\}$ of RosenbloomWidder. As in the case $\mu=1$, the solutions for $\mu>1$ are bi-orthogonal:

THEOREM 5.1. Define $W_{\mu}(r)=2 \pi^{\mu / 2} r^{\mu-1}$. For all $t>0, \mu \neq 2,4, \ldots$, we have

$$
\text { f.p. } \int_{0}^{\infty} V_{n}^{\mu}(r,-t) \tilde{V}_{m}^{\mu}(r, t) W_{\mu}(r) d r=\lambda_{m}^{\mu} \delta_{m, n}, \quad m, n=0,1, \ldots
$$


where

$$
\lambda_{m}^{\mu}=4^{2 m+2-\mu} m ! \Gamma(m+2-\mu / 2)
$$

For $\mu=2 k+2, k=0,1, \ldots$, the same result holds provided $m, n \geq k$.

Proof. From (2.3), (3.1), (3.4) and (3.6) we have

$$
\begin{aligned}
& \text { f.p. } \int_{0}^{\infty} V_{n}^{\mu}(r,-t) \tilde{V}_{m}^{\mu}(r, t) W_{\mu}(r) d r \\
& \quad=4^{n+m+2-\mu}(-1)^{n+m} n ! m ! t^{n-m} \text { f.p. } \int_{0}^{\infty} L_{n}^{(2-\mu) / 2}(u) L_{m}^{(2-\mu) / 2}(u) e^{-u} u^{(2-\mu) / 2} d u \\
& \quad=4^{2 m+2-\mu} m ! \Gamma(m+2-\mu / 2) \delta_{m, n} .
\end{aligned}
$$

The result for $\mu=2 k+2, k=0,1, \ldots$, also follows from (5.3) and (5.5), see $[1,4]$.

We obtain a generating function for $V_{m}^{\mu}(r, t)$ from their connection to the Laguerre polynomials. Since for all $\nu \in C([10])$,

$$
(1-t)^{-v-1} e^{-x t /(1-t)}=\sum_{k=0}^{\infty} L_{k}^{\nu}(x) t^{k}, \quad|t|<1,
$$

we have

$$
\begin{aligned}
\sum_{k=0}^{\infty} \frac{a^{k}}{k !} V_{k}^{\mu}(r, t) & =r^{2-\mu} \sum_{k=0}^{\infty} L_{k}^{(2-\mu) / 2}\left(-\frac{r^{2}}{4 t}\right)(4 a t)^{k} \\
& =r^{2-\mu}(1-4 a t)^{(\mu-4) / 2} e^{a r^{2} /(1-4 a t)}, \quad|a|<1 / 4 t
\end{aligned}
$$

By taking the Appell transform of both sides above we have

$$
(4 \pi r)^{2-\mu} k_{4-\mu}(r, t+4 a)=\sum_{k=0}^{\infty} \frac{a^{k}}{k !} \tilde{V}_{k}^{\mu}(r, t), \quad|a|<t / 4
$$

It is easily verified that

$$
\begin{gathered}
\frac{\partial}{\partial t} V_{m}^{\mu}(r, t)=2 m(2 m+2-\mu) V_{m-1}^{\mu}(r, t), \\
\frac{\partial}{\partial t} \tilde{V}_{k}^{\mu}(r, t)=\frac{1}{4} \tilde{V}_{k+1}^{\mu}(r, t) .
\end{gathered}
$$




\section{Regions of convergence for expansions}

A useful tool for estimating the size of coefficients in convergent expansions is:

FEJER'S FORMULA. ([10]) For all $v \in R$ and $x>0$

$$
L_{m}^{\nu}(x)=\pi^{-1 / 2} m^{v / 2-1 / 4} e^{x / 2} x^{-v / 2-1 / 4}\left(\cos \left\{2(m x)^{1 / 2}-(2 v+1) \pi / 4\right\}+\theta_{m, v}(x) / m^{1 / 2}\right)
$$

where $\theta_{m . v}(x)$ is uniformly bounded for $x \in[a, b], 0<a<b<\infty$, as $m \rightarrow \infty$.

From Fejer's formula, it follows easily as in [9, Lemma 5.2] that if $\sum_{m=0}^{\infty} a_{m} L_{m}^{\nu}(x)$ converges for all $x$ in some interval $[a, b], 0<a<b<\infty$, then

$$
a_{m}=o\left(m^{1 / 4-v / 2}\right), \quad m \rightarrow \infty .
$$

Therefore,

THEOREM 6.1. For $t=t_{0}<0, r^{2} \in[a, b], 0<a<b<\infty$, if

$$
\sum_{m=0}^{\infty} a_{m} V_{m}^{\mu}(r, t)
$$

converges, then

$$
a_{m}=o\left(\frac{m^{(\mu-1) / 4}}{\left(4 t_{0}\right)^{m} m !}\right), \quad m \rightarrow \infty
$$

LEMMA 6.2. For any $\delta>1$ and $r^{2}, \eta^{2}$ in a fixed interval $[a, b], 0<a<b<\infty$, we have as $m \rightarrow \infty$ :

$$
\begin{aligned}
V_{m}^{\mu}(r, t) & =O\left((4 t \delta)^{m} m !\right), & & t>0 \\
V_{m}^{\mu}(r, t) & =O\left((4|t|)^{m} m ! m^{(1-\mu) / 4}\right), & & t<0 \\
\tilde{V}_{m}^{\mu}(\eta, s) & =O\left((4 / s)^{m} m ! m^{(1-\mu) / 4}\right), & & s>0 \\
\tilde{V}_{m}^{\mu}(\eta, s) & =O\left((4 \delta /|s|)^{m} m !\right), & & s<0 .
\end{aligned}
$$

PROOF. This follows from Lemmas 4.5 and 4.6.

COROLLARY 6.3. If (6.3) converges for any $t_{0}<0, r^{2} \in[a, b], 0<a<b<\infty$, then it also converges for $|t|<\left|t_{0}\right|, r \neq 0$. Furthermore, $u(r, t)=\sum_{m=0}^{\infty} a_{m} V_{m}^{\mu}(r, t)$ defines a solution of (1.8) in $|t|<\left|t_{0}\right|$ such that $r^{\mu-2} u(r, t)$ is an entire function of $r^{2}$. 
PROOF. This follows from (6.4), (6.5), (6.6) and (5.10).

Note that if (6.3) converges for all $|t|<\sigma$ for some $\sigma>0$, then for any $t_{0} \in(0, \sigma)$ we have the simpler estimate

$$
a_{m}=o\left(\frac{1}{\left(4 t_{0}\right)^{m} m !}\right), \quad m \rightarrow \infty .
$$

THEOREM 6.4. If the series

$$
\sum_{m=0}^{\infty} a_{m} \tilde{V}_{m}^{\mu}(r, t)
$$

converges for some $t=t_{0}>0$ and $r^{2} \in[a, b], 0<a<b<\infty$, then

$$
a_{m}=o\left(\frac{m^{(\mu-1) / 4} t_{0}^{m}}{4^{m} m !}\right), \quad m \rightarrow \infty
$$

PROOF. The series (6.10) converges if and only if $\sum_{m=0}^{\infty} a_{m} t_{0}^{-2 m} V_{m}^{\mu}\left(r,-t_{0}\right)$ converges. Therefore (6.11) follows from (6.4).

COROLLARY 6.5. If the series (6.10) converges for some $t_{0}>0, r^{2} \in[a, b]$, then it also converges for $|t|>\left|t_{0}\right|$ and defines a solution $u(r, t)$ of $(1.8)$ there such that $r^{\mu-2} u(r, t)$ is entire in $r^{2}$.

\section{The double generating function}

Define the double generating function:

$$
S_{\mu}(r, \eta ; t, s)=\sum_{m=0}^{\infty} \frac{V_{m}^{\mu}(r, t) \tilde{V}_{m}^{\mu}(\eta, s)}{4^{2 m+2-\mu} m ! \Gamma\left(m+2-\frac{\mu}{2}\right)}, \quad|t|<|s| .
$$

The convergence of (7.1) follows from Lemma 6.2.

THEOREM 7.1. For all $r, \eta>0$ and $|t|<|s|$ we have

$$
S_{\mu}(r, \eta ; t, s)=\frac{(\eta r)^{2-\mu}}{\pi^{\mu / 2}(4(s+t))^{2-\mu / 2}} e^{-\left(r^{2}+\eta^{2}\right) / 4(s+t)} I_{(2-\mu) / 2}^{*}\left(\frac{r^{2} \eta^{2}}{16(s+t)^{2}}\right)
$$


PROOF. This follows from making the substitutions $v=(2-\mu) / 2, u=-t / s$, $x=-r^{2} / 4 t$, and $y=\eta^{2} / 4 s$ into ([8]):

$$
(1-u)^{-v-1} e^{-(x+y) u /(1-u)} I_{\nu}^{*}\left(\frac{x y u}{(1-u)^{2}}\right)=\sum_{m=0}^{\infty} \frac{m ! L_{m}^{v}(x) L_{m}^{v}(y)}{\Gamma(m+v+1)} u^{m}, \quad|u|<1 .
$$

Define for $s, r, \eta \neq 0$ :

$$
\begin{aligned}
K_{\mu}(r, \eta, s) & =\lim _{\substack{t \rightarrow 0+\\
}} S_{\mu}(r, \eta ; t, s) \\
& =\sum_{m=0}^{\infty} \frac{r^{2 m+2-\mu} \tilde{V}_{m}^{\mu}(\eta, s)}{4^{2 m+2-\mu} m ! \Gamma\left(m+2-\frac{\mu}{2}\right)} \\
& =\frac{(r \eta)^{2-\mu}}{\pi^{\mu / 2}(4 s)^{2-\frac{\mu}{2}}} e^{-\left(r^{2}+\eta^{2}\right) / 4 s} I_{\frac{2-\mu}{2}}^{*}\left(\frac{r^{2} \eta^{2}}{16 s^{2}}\right) .
\end{aligned}
$$

Note that for $\mu \neq 2 k+2, k=0,1, \ldots$, we have $K_{\mu}(r, \eta, s)=O\left((r \eta)^{2-\mu}\right), r \eta \rightarrow$ 0 . For $\mu=2 k+2, k=0,1, \ldots$,

$$
\begin{aligned}
K_{\mu}(r, \eta, s) & =\sum_{m=0}^{\infty} \frac{r^{2 m-2 k} \tilde{V}_{m}^{\mu}(\eta, s)}{4^{2 m-2 k} m ! \Gamma(m+1-k)} \\
& =\sum_{m=k}^{\infty} \frac{r^{2 m-2 k} \tilde{V}_{m}^{\mu}(\eta, s)}{4^{2 m-2 k} m ! \Gamma(m+1-k)} \\
& =\sum_{m=0}^{\infty} \frac{r^{2 m} \tilde{R}_{m}^{\mu}(\eta, s)}{4^{2 m} m ! \Gamma(m+1+k)}
\end{aligned}
$$

Since this coincides with the kernel given in [1, 4], and because of (5.3) and (5.5), from now on we consider only the case $\mu \in \Omega$, where we define

$$
\Omega=\{\mu: \mu>1, \mu \neq 2 k+2, k=0,1, \ldots\} .
$$

Note that

$$
\lim _{r \rightarrow 0+} r^{\mu-2} K_{\mu}(r, \eta, s)=\frac{(\pi \eta)^{2-\mu}}{\Gamma((4-\mu) / 2)} k_{4-\mu}(\eta, s) .
$$

From the asymptotic formula (4.6) we have as $r \eta / s \rightarrow \infty$

$$
K_{\mu}(r, \eta, s)=\frac{1}{\sqrt{4 \pi}} \frac{(\eta r)^{(1-\mu) / 2}}{\pi^{\mu / 2} \sqrt{4 s}}\left(e^{-(r-\eta)^{2} / 4 s}+e^{(3-\mu) \pi i / 2} e^{-(r+\eta)^{2} / 4 s}\right)\left\{1+O\left(\frac{s}{r \eta}\right)\right\} .
$$

Thus for fixed $s$,

$$
K_{\mu}(r, \eta, s)=O(1)(\eta r)^{(1-\mu) / 2} e^{-(r-\eta)^{2} / 4 s}, \quad r \eta \rightarrow \infty .
$$


We will also need estimates on the size of the derivatives of $K_{\mu}$. Define

$$
h_{\mu}(\xi, \eta)=e^{-(\xi+\eta)} I_{(2-\mu) / 2}^{*}(\xi \eta)
$$

Note that

$$
K_{\mu}(\xi, \eta, t)=\frac{(\xi \eta)^{2-\mu}}{\pi^{\mu / 2}(4 t)^{2-\mu / 2}} h_{\mu}\left(\frac{\xi^{2}}{4 t}, \frac{\eta^{2}}{4 t}\right)
$$

We have for $j=0,1, \ldots$

$$
\partial_{\xi}^{j} h_{\mu}(\xi, \eta)=(-1)^{j} e^{-(\xi+\eta)} \sum_{k=0}^{j}\left(\begin{array}{l}
j \\
k
\end{array}\right)(-\eta)^{k} I_{(2-\mu) / 2+k}^{*}(\xi \eta)
$$

so that by equation (4.19) we have

$$
\partial_{\xi}^{j} h_{\mu}(\xi, \eta)=O(1) \eta^{j}(\xi \eta)^{(\mu-3) / 4} e^{-(\sqrt{\xi}-\sqrt{\eta})^{2}}, \quad \xi \eta \rightarrow \infty .
$$

\section{The Huygens property.}

DEFINITION 8.1 (The Huygens property). Let $\mu>1$. Let $u(r, t)$ be a solution of the generalized heat equation (1.8) in a strip $a<t<b, r \neq 0$. We say that $u \in H^{*}(a, b)$ if and only if for $a<t^{\prime}<t<b$ we have

$$
r^{\mu-2} u(r, t)=\text { f.p. } \int_{0}^{\infty} r^{\mu-2} K_{\mu}\left(r, \xi, t-t^{\prime}\right) u\left(\xi, t^{\prime}\right) W_{\mu}(\xi) d \xi, \quad r \in R .
$$

Furthermore, if $u(r, t)$ is a solution of the generalized heat equation in the complement of such a strip, then we say $u(r, t) \in H^{*}(b, a)$ if and only if (8.1) holds whenever $b<t^{\prime}<t, t^{\prime}<t<a$ or both $t^{\prime}>b$ and $t<a$.

For $r=0$, this is understood to mean that

$$
\lim _{r \rightarrow 0+} r^{\mu-2} u(r, t)=\frac{\pi^{2-\mu}}{\Gamma((4-\mu) / 2)} \text { f.p. } \int_{0}^{\infty} k_{4-\mu}\left(\xi, t-t^{\prime}\right) u\left(\xi, t^{\prime}\right) \xi^{2-\mu} W_{\mu}(\xi) d \xi ;
$$

see equation (7.6).

Note that the existence of the integral in (8.2) implies that, for any $c>0$,

$$
\int_{c}^{\infty} e^{-\xi^{2} / 4\left(t-t^{\prime}\right)}\left|u\left(\xi, t^{\prime}\right)\right| \xi d \xi<\infty, \quad a<t^{\prime}<t<b .
$$


LEMMA 8.2. If $u(r, t) \in H^{*}(-\sigma, \sigma)$ then $r^{\mu-2} u(r, t)$ is an entire function of $r^{2}$ for each $t \in(a, b)$.

PROOF. This follows easily from Theorem 4.4.

THEOREM 8.3. For all $\mu \in \Omega, m=0,1, \ldots$, we have

$$
V_{m}^{\mu}(r, t) \in H^{*}(-\infty, \infty) \text {. }
$$

PROOF. The identity

$$
r^{\mu-2} V_{m}^{\mu}(r, t)=\text { f.p. } \int_{0}^{\infty} r^{\mu-2} K_{\mu}\left(r, \xi, t-t^{\prime}\right) V_{m}^{\mu}\left(\xi, t^{\prime}\right) W_{\mu}(\xi) d \xi, \quad t^{\prime}<t, r \in R
$$

for $t \neq 0$ follows from letting

$$
v=\frac{2-\mu}{2}, \quad a=\frac{t^{\prime}-t}{t^{\prime}}, \quad x=\frac{r^{2}}{4\left(t-t^{\prime}\right)}, \quad \text { and } \quad y=\frac{\xi^{2}}{4\left(t-t^{\prime}\right)}
$$

in Theorem 4.2. The case $t=0$ follows from making the same substitutions into the equality of Corollary 4.3 .

COROLLARY 8.4. For $m=0,1, \ldots, \mu \in \Omega$,

$$
r^{\mu-2} V_{m}^{\mu}(r, t)=\text { f.p. } \int_{0}^{\infty} r^{\mu-2} K_{\mu}(r, \xi, t) \xi^{2 m+2-\mu} W_{\mu}(\xi) d \xi, \quad t>0, r \in R .
$$

THEOREM 8.5. For every generalized temperature function $u(r, t)$ and every $\sigma>0$, we have $u(r, t) \in H^{*}(-\sigma, \sigma)$ if and only if $\mathscr{A}_{\mu} u(r, t) \in H^{*}(1 / \sigma,-1 / \sigma)$.

PROOF. Assume $r \neq 0$. If $u \in H^{*}(-\sigma, \sigma)$ then for $-\sigma<-1 / t^{\prime}<t<\sigma$ we have

$$
u(r, t)=\text { f.p. } \int_{0}^{\infty} K_{\mu}\left(r, \xi, t+\frac{1}{t^{\prime}}\right) u\left(\xi,-\frac{1}{t^{\prime}}\right) W_{\mu}(\xi) d \xi .
$$

Therefore, if $-\sigma<-1 / t^{\prime}<-1 / t<\sigma$, or equivalently if $1 / \sigma<t^{\prime}<t, t^{\prime}<t<$ $-1 / \sigma$ or both $t^{\prime}>1 / \sigma$ and $t<-1 / \sigma$, we have

$$
\begin{aligned}
\mathscr{A}_{\mu} u(r, t) & =k_{\mu}(r, t) \text { f.p. } \int_{0}^{\infty} K_{\mu}\left(\frac{r}{t}, \xi, \frac{1}{t^{\prime}}-\frac{1}{t}\right) u\left(\xi,-\frac{1}{t^{\prime}}\right) W_{\mu}(\xi) d \xi \\
& =k_{\mu}(r, t) \text { f.p. } \int_{0}^{\infty} K_{\mu}\left(\frac{r}{t}, \frac{\xi}{t^{\prime}}, \frac{1}{t^{\prime}}-\frac{1}{t}\right) u\left(\frac{\xi}{t^{\prime}},-\frac{1}{t^{\prime}}\right) W_{\mu}\left(\frac{\xi}{t^{\prime}}\right) d\left(\frac{\xi}{t^{\prime}}\right) \\
& =\frac{k_{\mu}(r, t)}{\left(t^{\prime}\right)^{\mu}} \text { f.p. } \int_{0}^{\infty} K_{\mu}\left(\frac{r}{t}, \frac{\xi}{t^{\prime}}, \frac{1}{t^{\prime}}-\frac{1}{t}\right) \frac{1}{k_{\mu}\left(\xi, t^{\prime}\right)} \mathscr{A}_{\mu} u\left(\xi, t^{\prime}\right) W_{\mu}(\xi) d \xi \\
& =\text { f.p. } \int_{0}^{\infty} K_{\mu}\left(r, \xi, t-t^{\prime}\right) \mathscr{A}_{\mu} u\left(\xi, t^{\prime}\right) W_{\mu}(\xi) d \xi .
\end{aligned}
$$


For $r=0$, note that

$$
\begin{aligned}
\lim _{r \rightarrow 0} r^{\mu-2} \mathscr{A}_{\mu} u(r, t) & =\lim _{r \rightarrow 0} r^{\mu-2} u\left(\frac{r}{t}, \frac{-1}{t}\right) k_{\mu}(r, t) \\
& =t^{\mu-2} k_{\mu}(0, t) \lim _{r \rightarrow 0} r^{\mu-2} u(r,-1 / t)
\end{aligned}
$$

and the rest of the proof follows as before.

The proof in the other direction is similar.

THEOREM 8.6. For $\mu \in \Omega, m=0,1, \ldots$, we have

$$
\tilde{V}_{m}^{\mu}(r, t) \in H^{*}(0+, 0-) .
$$

PROOF. This follows from Theorems 8.3 and 8.5.

THEOREM 8.7. For $0<t^{\prime}<t, t^{\prime}<t<0$ or both $t^{\prime}>0$ and $t<0$ we have

$$
\begin{aligned}
& r^{\mu-2} K_{\mu}(r, \eta, t)=\text { f.p. } \int_{0}^{\infty} r^{\mu-2} K_{\mu}\left(r, \xi, t-t^{\prime}\right) K_{\mu}\left(\xi, \eta, t^{\prime}\right) W_{\mu}(\xi) d \xi \\
& r \in R, \eta \neq 0 .
\end{aligned}
$$

ProOF. Let

$$
C\left(r, \eta, t, t^{\prime}\right)=\frac{\eta^{2-\mu} e^{-r^{2} / 4\left(t-t^{\prime}\right)} e^{-\eta^{2} / 4 t^{\prime}}}{\left(16 t^{\prime}\left(t-t^{\prime}\right)\right)^{2-\mu / 2}}
$$

Since

$$
\begin{aligned}
& \text { f.p. } \int_{0}^{\infty} r^{\mu-2} K_{\mu}\left(r, \xi, t-t^{\prime}\right) K_{\mu}\left(\xi, \eta, t^{\prime}\right) W_{\mu}(\xi) d \xi \\
& =2 C\left(r, \eta, t, t^{\prime}\right) \text { f.p. } \int_{0}^{\infty} e^{-\xi^{2}\left(1 / 4\left(t-t^{\prime}\right)+1 / 4 t^{\prime}\right)} I_{(2-\mu) / 2}^{*}\left(\frac{r^{2} \xi^{2}}{16\left(t-t^{\prime}\right)^{2}}\right) \\
& \quad I_{(2-\mu) / 2}^{*}\left(\frac{\eta^{2} \xi^{2}}{16\left(t^{\prime}\right)^{2}}\right) \xi^{3-\mu} d \xi \\
& =C\left(r, \eta, t, t^{\prime}\right) \text { f.p. } \int_{0}^{\infty} e^{-\xi\left(1 / 4\left(t-t^{\prime}\right)+1 / 4 t^{\prime}\right)} I_{(2-\mu) / 2}^{*}\left(\frac{r^{2} \xi}{16\left(t-t^{\prime}\right)^{2}}\right) \\
& I_{(2-\mu) / 2}^{*}\left(\frac{\eta^{2} \xi}{16\left(t^{\prime}\right)^{2}}\right) \xi^{(2-\mu) / 2} d \xi
\end{aligned}
$$

we obtain the result from Corollary 4.9. 


\section{The Huygens Property of Poisson integrals}

THEOREM 9.1. Let $1<\mu<4$. Define $u(r, t)$ by

$(9.1)$

$$
r^{\mu-2} u(r, t)=\int_{0}^{\infty} r^{\mu-2} K_{\mu}(r, \xi, t-a) G(\xi) W_{\mu}(\xi) d \xi, \quad a<t<b, r \in R,
$$

where we assume that the integral converges absolutely for $r \in R$. Then

$$
u(r, t) \in H^{*}(a, b) .
$$

PROOF. For $1<\mu<4$ we note that $K_{\mu}(r, \xi, t)$ is non-negative for non-negative values of the arguments. Thus if $G \geq 0$, then for all $r \in R, a<t^{\prime}<t<b$, we have

$$
\begin{aligned}
\int_{0}^{\infty} & r^{\mu-2} K_{\mu}\left(r, \xi, t-t^{\prime}\right) u\left(\xi, t^{\prime}\right) W_{\mu}(\xi) d \xi \\
& =\int_{0}^{\infty} r^{\mu-2} K_{\mu}\left(r, \xi, t-t^{\prime}\right)\left(\int_{0}^{\infty} K_{\mu}\left(\xi, \eta, t^{\prime}-a\right) G(\eta) W_{\mu}(\eta) d \eta\right) W_{\mu}(\xi) d \xi \\
& =\int_{0}^{\infty}\left(\int_{0}^{\infty} r^{\mu-2} K_{\mu}\left(r, \xi, t-t^{\prime}\right) K_{\mu}\left(\xi, \eta, t^{\prime}-a\right) W_{\mu}(\xi) d \xi\right) G(\eta) W_{\mu}(\eta) d \eta \\
& =\int_{0}^{\infty} r^{\mu-2} K_{\mu}(r, \eta, t-a) G(\eta) W_{\mu}(\eta) d \eta \\
& =r^{\mu-2} u(r, t),
\end{aligned}
$$

where the exchange of integration is justified by Tonelli's theorem. For arbitrary $G$, write $G$ as the difference of its positive and negative parts, and the theorem follows from the case $G \geq 0$.

THEOREM 9.2. Suppose $-(n+1)<(2-\mu) / 2<-n, n \in N$. Define $u(r, t)$ by (9.4)

$$
r^{\mu-2} u(r, t)=\text { f.p. } \int_{0}^{\infty} r^{\mu-2} K_{\mu}(r, \xi, t-a) G(\xi) W_{\mu}(\xi) d \xi, \quad a<t<b, r \in R
$$

where $G(\xi)=\xi^{2-\mu} F\left(\xi^{2}\right), F(\xi)$ has $n$ continuous derivatives in some interval $[0, c]$, $c>0$, and

$$
\int_{c}^{\infty}\left|K_{\mu}(r, \xi, t-a) G(\xi)\right| W_{\mu}(\xi) d \xi<\infty .
$$

Then $u(r, t) \in H^{*}(a, b)$. 
PROOF. Formally, as in Theorem 9.1, the result follows from the interchange of integration. The justification will follow from Theorem 2.3. We may assume $a=0$, so that $0<t^{\prime}<t<b$. Using the change of variables $\eta^{2} \rightarrow \eta$, $\xi^{2} \rightarrow \xi$ we may rewrite (9.3) as:

$$
\frac{1}{\left(16 t^{\prime}\left(t-t^{\prime}\right)\right)^{2-\mu / 2}} \text { f.p. } \int_{0}^{\infty}\left(\text { f.p. } \int_{0}^{\infty} f(\xi, \eta)(\xi \eta)^{(2-\mu) / 2} d \xi\right) d \eta
$$

where

$$
f(\xi, \eta)=h_{\mu}\left(\frac{\xi}{4\left(t-t^{\prime}\right)}, \frac{r^{2}}{4\left(t-t^{\prime}\right)}\right) h_{\mu}\left(\frac{\xi}{4 t^{\prime}}, \frac{\eta}{4 t^{\prime}}\right) F(\eta)
$$

see equation (7.10). Firstly, note that $f(\xi, \eta)$ has continuous mixed partials up to degree $n$ in each variable in $[0, c] \times[0, c]$.

Secondly, since by equations (4.21), (7.9), and (7.12) with $j=0$,

$$
h_{\mu}\left(\frac{\xi}{4 t}, \frac{\eta}{4 t}\right)=O(1)(\xi \eta)^{(\mu-3) / 4} e^{-\eta / 4 \delta t} e^{\xi / 4(\delta-1) t}, \quad \xi \eta \rightarrow \infty,
$$

we have

$$
\begin{aligned}
\int_{c}^{\infty} \int_{c}^{\infty} & |f(\xi, \eta)|(\xi \eta)^{(2-\mu) / 2} d \xi d \eta \\
= & O(1) \int_{c}^{\infty} e^{-\eta / 4 \delta t^{\prime}}|F(\eta)| \eta^{(1-\mu) / 4} d \eta \\
& \cdot \int_{c}^{\infty} e^{\xi / 4(\delta-1) t^{\prime}}\left|h_{\mu}\left(\frac{\xi}{4\left(t-t^{\prime}\right)}, \frac{r^{2}}{4\left(t-t^{\prime}\right)}\right)\right| \xi^{(1-\mu) / 4} d \xi
\end{aligned}
$$

which is finite provided $\delta \in\left(t / t^{\prime}, b / t^{\prime}\right)$.

Next, note that

$$
\partial_{\xi}^{j} f(\xi, \eta)=F(\eta) \sum_{k=0}^{j}\left(\begin{array}{l}
j \\
k
\end{array}\right)\left(\partial_{\xi}^{k} h_{\mu}\left(\frac{\xi}{4\left(t-t^{\prime}\right)}, \frac{r^{2}}{4\left(t-t^{\prime}\right)}\right)\right)\left(\partial_{\xi}^{j-k} h_{\mu}\left(\frac{\xi}{4 t^{\prime}}, \frac{\eta}{4 t^{\prime}}\right)\right)
$$

so that since $I_{v}^{*}$ is of exponential growth $1 / 2$, given $\delta>1$, we have uniformly for $\xi \in[0, c]:$

$$
\left|\partial_{\xi}^{j} f(\xi, \eta)\right|=O(1)|F(\eta)| e^{-\eta / 4 \delta t^{\prime}}, \quad \eta \rightarrow \infty .
$$

This implies conditions (2.7) and (2.9) of Theorem 2.3.

A similar estimate can be found for $\left|\partial_{\eta}^{j} f(\xi, \eta)\right|$ for $\eta \in[0, c], \xi \rightarrow \infty$. This completes the proof. 


\section{Expansions and the Huygens property}

THEOREM 10.1. If

$$
u(r, t)=\sum_{m=0}^{\infty} a_{m} V_{m}^{\mu}(r, t)
$$

converges for $|t|<\sigma, r \neq 0$, then

$$
u(r, t) \in H^{*}(-\sigma, \sigma) .
$$

PROOF. Formally, we have using Theorem 8.3

$$
\begin{aligned}
\text { f.p. } \int_{0}^{\infty} u\left(\xi, t^{\prime}\right) r^{\mu-2} K_{\mu}\left(r, \xi, t-t^{\prime}\right) W_{\mu}(\xi) d \xi \\
=\text { f.p. } \int_{0}^{\infty}\left(\sum_{m=0}^{\infty} a_{m} V_{m}^{\mu}\left(\xi, t^{\prime}\right)\right) r^{\mu-2} K_{\mu}\left(r, \xi, t-t^{\prime}\right) W_{\mu}(\xi) d \xi \\
=\sum_{m=0}^{\infty} a_{m} \text { f.p. } \int_{0}^{\infty} V_{m}^{\mu}\left(\xi, t^{\prime}\right) r^{\mu-2} K_{\mu}\left(r, \xi, t-t^{\prime}\right) W_{\mu}(\xi) d \xi \\
=\sum_{m=0}^{\infty} a_{m} r^{\mu-2} V_{m}^{\mu}(r, t)=r^{\mu-2} u(r, t) .
\end{aligned}
$$

Let $c_{m}=a_{m}\left(4 t^{\prime}\right)^{m} m$ !. By equation (6.9), $c_{m}=O\left(\left(t^{\prime} / t_{0}\right)^{m}\right)$ for any $t_{0} \in(0, \sigma)$. Using the change of variables $\xi^{2} \rightarrow-4 t^{\prime} \xi$ the sum in (10.3) may be rewritten as

$$
\left(\frac{-t^{\prime}}{t-t^{\prime}}\right)^{2-\mu / 2} \sum_{m=0}^{\infty} c_{m} \text { f.p. } \int_{0}^{\infty} L_{m}^{(2-\mu) / 2}(\xi) h_{\mu}\left(\frac{-t^{\prime} \xi}{t-t^{\prime}}, \frac{r^{2}}{4\left(t-t^{\prime}\right)}\right) \xi^{(2-\mu) / 2} d \xi .
$$

It is easy to verify that for any $c>0$ and $t^{\prime}<t<-t^{\prime}, t^{\prime} \in(-\sigma, 0)$ we have:

$$
\int_{c}^{\infty}\left|h_{\mu}\left(\frac{-t^{\prime} \xi}{t-t^{\prime}}, \frac{r^{2}}{4\left(t-t^{\prime}\right)}\right)\right| e^{\xi / 2} \xi^{(1-\mu) / 4} d \xi<\infty .
$$

Thus the interchange of summation and integration is justified for $t^{\prime}<t<-t^{\prime}$ by Theorem 4.7.

Therefore, Theorems 9.1 and 9.2 imply that $u(r, t) \in H^{*}\left(t^{\prime},-t^{\prime}\right)$, and since $t^{\prime}$ can be chosen arbitrarily close to $-\sigma$ this proves the theorem.

LEMMA 10.2. If for $\sigma>0, u \in H^{*}(-\sigma, \sigma)$ then

$$
\text { f.p. } \int_{0}^{\infty} \tilde{V}_{m}^{\mu}(\xi,-t) u(\xi, t) W_{\mu}(\xi) d \xi
$$

exists and is independent of $t \in(-\sigma, 0)$. 
ProOF. Let $-\sigma<t^{\prime}<t<0$. Formally, we have

(10.4)

$$
\begin{aligned}
& \text { f.p. } \int_{0}^{\infty} \tilde{V}_{m}^{\mu}(\xi,-t) u(\xi, t) W_{\mu}(\xi) d \xi \\
& \quad=\text { f.p. } \int_{0}^{\infty} \tilde{V}_{m}^{\mu}(\xi,-t)\left(\text { f.p. } \int_{0}^{\infty} u\left(\eta, t^{\prime}\right) K_{\mu}\left(\xi, \eta, t-t^{\prime}\right) W_{\mu}(\eta) d \eta\right) W_{\mu}(\xi) d \xi \\
& \quad=\text { f.p. } \int_{0}^{\infty} u\left(\eta, t^{\prime}\right)\left(\text { f.p. } \int_{0}^{\infty} K_{\mu}\left(\xi, \eta, t-t^{\prime}\right) \tilde{V}_{m}^{\mu}(\xi,-t) W_{\mu}(\xi) d \xi\right) W_{\mu}(\eta) d \eta \\
& \quad=\text { f.p. } \int_{0}^{\infty} \tilde{V}_{m}^{\mu}\left(\eta,-t^{\prime}\right) u\left(\eta, t^{\prime}\right) W_{\mu}(\eta) d \eta .
\end{aligned}
$$

We now justify the exchange of integration.

By Lemma 8.2, we can write $u(\xi, t)=\xi^{2-\mu} v\left(\xi^{2}, t\right)$ where $v(\xi, t)$ is entire in the first variable. By the change of variables $\eta^{2} \rightarrow \eta$ and $\xi^{2} \rightarrow \xi$, the integral in (10.4) can be written

$$
\frac{m ! 4^{m-2}}{(-t)^{m+2-\mu / 2}\left(t-t^{\prime}\right)^{2-\mu / 2}} \text { f.p. } \int_{0}^{\infty}\left(\text { f.p. } \int_{0}^{\infty} f(\xi, \eta)(\xi \eta)^{(2-\mu) / 2} d \xi\right) d \eta
$$

where

$$
f(\xi, \eta)=v\left(\eta, t^{\prime}\right) L_{m}^{(2-\mu) / 2}\left(-\frac{\xi}{4 t}\right) e^{\xi / 4 t} h_{\mu}\left(\frac{\xi}{4\left(t-t^{\prime}\right)}, \frac{\eta}{4\left(t-t^{\prime}\right)}\right) .
$$

We will justify the interchange of integration using Theorem 2.3. Fix $c>0$. Firstly, note that $f(\xi, \eta)$ is entire in $\xi$ and $\eta$.

Secondly, using equations (4.21) and (7.12) with $j=0$ we have

$$
\begin{aligned}
& \int_{c}^{\infty} \int_{c}^{\infty}|f(\xi, \eta)|(\xi \eta)^{(2-\mu) / 2} d \xi d \eta \\
&=O(1) \int_{c}^{\infty}\left|v\left(\eta, t^{\prime}\right)\right| e^{-\eta / 4 \delta\left(t-t^{\prime}\right)} \eta^{(1-\mu) / 4} d \eta \\
& \cdot \int_{c}^{\infty}\left|L_{m}^{(2-\mu) / 2}(-\xi / 4 t)\right| e^{\xi / 4 t} e^{\xi / 4(\delta-1)\left(t-t^{\prime}\right)} \xi^{(1-\mu) / 4} d \xi
\end{aligned}
$$

which is finite provided $\delta-1 \in\left(-t /\left(t-t^{\prime}\right),(\sigma-t) /\left(t-t^{\prime}\right)\right)$; see equation (8.3).

Finally, since

$$
\partial_{\eta}^{j} f(\xi, \eta)=L_{m}^{(2-\mu) / 2}\left(\frac{-\xi}{4 t}\right) e^{\xi / 4 t} \sum_{k=0}^{j}\left(\begin{array}{l}
j \\
k
\end{array}\right)\left(\partial_{\eta}^{k} v(\eta, t)\right)\left(\partial_{\eta}^{j-k} h_{\mu}\left(\frac{\xi}{4\left(t-t^{\prime}\right)}, \frac{\eta}{4\left(t-t^{\prime}\right)}\right)\right)
$$

given $\delta>1$ we have uniformly for $\eta \in[0, c]$,

$$
\left|\partial_{\eta}^{j} f(\xi, \eta)\right|=O(1)\left|L_{m}^{(2-\mu) / 2}(-\xi / 4 t)\right| e^{\xi / 4 t} e^{-\xi / 4 \delta\left(t-t^{\prime}\right)}, \quad \xi \rightarrow \infty
$$


so that conditions (2.6) and (2.8) of Theorem 2.3 are satisfied. A similar estimate holds for $\xi \in[0, c]$ and $\eta \rightarrow \infty$.

This completes the proof.

THEOREM 10.3. If for $\sigma>0, u \in H^{*}(-\sigma, \sigma)$, then $u(r, t)$ has an expansion $u(r, t)=\sum_{m=0}^{\infty} a_{m} V_{m}^{\mu}(r, t)$ converging pointwise for $-\sigma<t<\sigma, r \neq 0$, with $a_{m}=\frac{1}{4^{2 m+2-\mu} m ! \Gamma(m+2-\mu / 2)} f \cdot p \cdot \int_{0}^{\infty} \tilde{V}_{m}^{\mu}(\xi,-t) u(\xi, t) W_{\mu}(\xi) d \xi, \quad-\sigma<t<0$.

PROOF. From Lemma 10.2 the representation of $a_{m}$ is independent of $t$. Since for $|t|<\left|t^{\prime}\right|:$

$$
K_{\mu}\left(r, \xi, t-t^{\prime}\right)=\sum_{m=0}^{\infty} \frac{V_{m}^{\mu}(r, t) \tilde{V}_{m}^{\mu}\left(\xi,-t^{\prime}\right)}{4^{2 m+2-\mu} m ! \Gamma\left(m+2-\frac{\mu}{2}\right)}
$$

we have formally for $-\sigma<t^{\prime}<0, t^{\prime}<t<-t^{\prime}, r \neq 0$

$$
\begin{aligned}
u(r, t) & =\sum_{m=0}^{\infty} \frac{V_{m}^{\mu}(r, t)}{4^{2 m+2-\mu} m ! \Gamma\left(m+2-\frac{\mu}{2}\right)} \mathrm{f} . \mathrm{p} \cdot \int_{0}^{\infty} \tilde{V}_{m}^{\mu}\left(\xi,-t^{\prime}\right) u\left(\xi, t^{\prime}\right) W_{\mu}(\xi) d \xi \\
& =\sum_{m=0}^{\infty} a_{m} V_{m}^{\mu}(r, t) .
\end{aligned}
$$

Writing $u(\xi, t)=\xi^{2-\mu} v\left(\xi^{2}, t\right), v(\xi, t)$ entire in $\xi$, we may rewrite (10.5) as

$$
\begin{aligned}
& \frac{1}{4^{2-\mu}\left(-4 t^{\prime}\right)^{2-\mu / 2}} \sum_{m=0}^{\infty} \frac{V_{m}^{\mu}(r, t)}{\left(4 t^{\prime}\right)^{m} \Gamma\left(m+2-\frac{\mu}{2}\right)} \\
& \text { f.p. } \int_{0}^{\infty} e^{\xi / 4 t^{\prime}} L_{m}^{(2-\mu) / 2}\left(-\frac{\xi}{4 t^{\prime}}\right) v\left(\xi, t^{\prime}\right) \xi^{(2-\mu) / 2} d \xi .
\end{aligned}
$$

By (8.3) with $t=-t^{\prime}$ we have

$$
\int_{c}^{\infty} e^{\xi / 8 t^{\prime}}\left|v\left(\xi, t^{\prime}\right)\right| \xi^{(1-\mu) / 4} d \xi<\infty
$$

Thus by Theorem 4.7 and Lemma 6.2 we obtain the result.

THEOREM 10.4. If for $\sigma>0, u(r, t)=\sum_{m=0}^{\infty} a_{m} \tilde{V}_{m}^{\mu}(r, t)$ converges pointwise for $|t|>\sigma$ and $r \neq 0$ then $u(r, t) \in H^{*}(\sigma,-\sigma)$. 
PROOF. Since

$$
\mathscr{A}_{\mu} u(r, t)=\frac{e^{-3 \pi \mu i / 2}}{(4 \pi)^{\mu}} \sum_{m=0}^{\infty} a_{m} V_{m}^{\mu}(r, t)
$$

converges in $|t|<1 / \sigma$, Theorem 10.1 implies that $\mathscr{A}_{\mu} u \in H^{*}(-1 / \sigma, 1 / \sigma)$. Thus by Theorem 8.5 we have $u \in H^{*}(\sigma,-\sigma)$.

THEOREM 10.5. If for $\sigma>0, u(r, t) \in H^{*}(\sigma,-\sigma)$ then $u(r, t)=\sum_{m=0}^{\infty} a_{m} \tilde{V}_{m}^{\mu}(r, t)$ where

$$
a_{m}=\frac{1}{4^{2 m+2-\mu} m ! \Gamma\left(m+2-\frac{\mu}{2}\right)} \text { f.p. } \int_{0}^{\infty} V_{m}^{\mu}(\xi, t) u(\xi,-t) W_{\mu}(\xi) d \xi, \quad t<-\sigma .
$$

Proof. If $u \in H^{*}(\sigma,-\sigma)$, then $\mathscr{A}_{\mu} u(r, t) \in H^{*}(-1 / \sigma, 1 / \sigma)$. Thus by Theorem $10.3, \mathscr{A}_{\mu} u(r, t)=\sum_{m=0}^{\infty} b_{m} V_{m}^{\mu}(r, t),|t|<1 / \sigma$, where for $-1 / \sigma<t<0$

$$
b_{m}=\frac{1}{4^{2 m+2-\mu} m ! \Gamma\left(m+2-\frac{\mu}{2}\right)} \text { f.p. } \int_{0}^{\infty} \tilde{V}_{m}^{\mu}(\xi,-t) \mathscr{A}_{\mu} u(\xi, t) W_{\mu}(\xi) d \xi .
$$

Since $\tilde{V}_{m}^{\mu}(\xi,-t)=k_{\mu}(\xi,-t) V_{m}^{\mu}(-\xi / t, 1 / t)$ and $\mathscr{A}_{\mu} u(\xi, t)=k_{\mu}(\xi, t) u(\xi / t,-1 / t)$ we have

$$
\begin{aligned}
& \text { f.p. } \int_{0}^{\infty} \tilde{V}_{m}^{\mu}(\xi,-t) \mathscr{A}_{\mu} u(\xi, t) W_{\mu}(\xi) d \xi \\
& =\frac{e^{-3 \pi i \mu / 2}}{(4 \pi)^{\mu}} \text { f.p. } \int_{0}^{\infty} V_{m}^{\mu}\left(\xi, \frac{1}{t}\right) u\left(\xi,-\frac{1}{t}\right) W_{\mu}(\xi) d \xi
\end{aligned}
$$

so that

$$
\begin{aligned}
b_{m} & =\frac{e^{-3 \pi i \mu / 2}}{(4 \pi)^{\mu}} \frac{1}{4^{2 m+2-\mu} m ! \Gamma\left(m+2-\frac{\mu}{2}\right)} \text { f.p. } \int_{0}^{\infty} V_{m}^{\mu}(\xi, t) u(\xi,-t) W_{\mu}(\xi) d \xi, \\
t & <-\sigma
\end{aligned}
$$

Therefore, since $u(r, t)=(4 \pi)^{\mu} \mathscr{A}_{\mu}^{2} u(r, t) / e^{-3 \pi i \mu / 2}$, we obtain the result.

\section{References}

[1] L. R. Bragg, 'Radial heat polynomials and related functions', Trans. Amer. Math. Soc. 119 (1965), 270-290.

[2] A. Erdelyi, Quart. J. Math. Oxford Ser. (2) 9 (1938), 196-198. 
[3] J. Hadamard, Lectures on Cauchy's problem in linear partial differential equations (Dover, New York, 1952).

[4] D. T. Haimo, 'Expansions in terms of generalized heat polynomials and of their Appell transforms', J. Appl. Math. Mech. 15 (1966), 735-758.

[5] — 'Homogeneous generalized temperatures', SIAM J. Math. Anal. 11 (1980), 473-478.

[6] E. Kochneff and Y. Sagher, 'The Appell transform and the semigroup property for temperatures', J. Austral. Math. Soc. (Series A) 60 (1996), 109-117.

[7] A. Krall and R. Morton, 'Distributional weight functions for orthogonal polynomials', SIAM J. Math. Anal. 9 (1978), 604-626.

[8] N. N. Lebedev, Special functions and their applications (Prentice-Hall, New Jersey, 1965).

[9] P. C. Rosenbloom and D. V. Widder, 'Expansions in terms of heat polynomials and associated functions', Trans. Amer. Math. Soc. 92 (1959), 604-626.

[10] G. Szego, Orthogonal polynomials, Amer. Math. Soc. Colloq. Pub. 23 (Amer. Math. Soc., Providence, 1979).

\section{Department of Mathematics}

Eastern Washington University

Cheney WA 99004

USA

e-mail: ekochneff@ewu.edu 